$62^{\text {ème }}$ Congrès de la SFCO, 03006 (2014)

DOI: $10.1051 /$ sfco/20146203006

(C) Owned by the authors, published by EDP Sciences, 2014

\title{
Intérêt de la vaccination par voie muqueuse dans la prise en charge des cancers des Voies Aéro-digestives supérieures (VADS).
}

\author{
Lescaille $\mathbf{G}^{1-2}$, Macedo $\mathbf{R}^{1}$, Baillou $\mathbf{C}^{\mathbf{1}}$, Noizat $\mathbf{C}^{\mathbf{1}}$, Bertolus $\mathbf{C}^{\mathbf{1}}$, Bellier $\mathbf{B}^{1}$, Mateo $\mathbf{V}^{\mathbf{1}}$, Lemoine $\mathrm{F}^{1}$. \\ 1 UPMC Paris 06, CIMI inserm U1135, Groupe hospitalier Pitié-Salpêtrière \\ 2 Université Denis Diderot Paris 07
}

Les cancers des VADS représentent un problème de santé publique majeur compte tenu de leur fréquence, et se situent actuellement à la troisième place masculine française. Leurs principaux facteurs de risque sont les intoxications tabagique et alcoolique, notamment en association. Toutefois, depuis quelques années, il a été montré que le papillomavirus de type 16 (HPV16), connu pour être à l'origine de cancers ano-génitaux, pouvaient également provoquer des cancers de l'oropharynx (amygdale et base de langue). Bien que les connaissances concernant leur développement aient beaucoup évoluées, le pronostic de ces cancers reste sombre malgré les thérapeutiques actuelles. II est donc important de développer de nouvelles thérapeutiques et la vaccination thérapeutique constitue une piste intéressante. Parce que les cancers des VADS se développent au niveau de muqueuses et ont une forte tendance aux récidives loco-régionales, il est important d'utiliser des voies vaccinales favorisant des réponses muqueuses.

Nous avons développé un modèle orthotopique de cancer de la cavité orale chez la souris C57/BL6 en injectant des cellules épithéliales cancéreuses issues d'une lignée murine appelée TC1 au niveau de la joue. Nous avons ensuite comparé l'efficacité d'une vaccination précédemment décrite (Lescaille et al. 2013), utilisant une stratégie ADN destinée à produire des pseudo-particules virales (VLP) exprimant l'onco-protéine E7 de l'HPV16 en association avec des adjuvants. On parle ainsi de plasmo-retro VLPS (pVLPS) du fait que les VLPs sont produites in vivo après l'administration d'un vecteur plasmidique contenant les séquences nécessaires et suffisantes à l'expression des protéines constitutives des VLPs qui sont ensuite relarguées dans l'environnement extracellulaire. Les VLPs ainsi produites in vivo ont une meilleure immunogénicité que des protéines solubles. Cette stratégie qui présente l'avantage d'une production simple, rapide, peu onéreuse, a pour but d'entraîner des réponses anti-tumorales en entraînant une réponse immune forte contre les cellules exprimant cet antigène. Nous avons ainsi comparé l'efficacité des voies de vaccination intra-nasale, intra-dermique (ID) et intra-muqueuse (IJ, dans la joue), tant au niveau des réponses immunes locales et systémiques, que des réponses anti-tumorales. Nous avons étudié les réponses T CD8 spécifique d'E7 par technique ELIspot IFNy et par tétramère, au niveau du sang, de la tumeur, et des ganglions. Nous avons mesuré le volume tumoral au pied à coulisse.

Dans un contexte de tumeurs établies, nous avons montré que la vaccination muqueuse jugale permettait d'obtenir de meilleures réponses anti-tumorales que la vaccination intradermique, avec ou sans adjuvant, et que ces réponses étaient améliorées dans les deux groupes (IJ et ID) avec des adjuvants. Par ailleurs, nous avons observé que les réponses immunes étaient meilleures en association avec des adjuvants, et que les réponses locales (ganglion et tumeur) induites par une vaccination muqueuse intrajugale étaient plus importantes qu'avec une vaccination intradermique (augmentation des CD8 et des CD8 spécifiques d'E7). Ces résultats expérimentaux suggèrent que la voie vaccinale intra-muqueuse serait plus efficace pour traiter des tumeurs de la cavité orale et pourrait induire, du fait de meilleures réponses immunitaires loco-régionales, une meilleure protection contre les récidives.

This is an Open Access article distributed under the terms of the Creative Commons Attribution License 4.0, which permits unrestricted use, distribution, and reproduction in any medium, provided the original work is properly cited. 